\title{
ON SOME BOUNDS OF THE MINIMUM EDGE DOMINATING ENERGY OF A GRAPH
}

\author{
A.Sharmila ${ }^{a}$, S. Lavanya ${ }^{b}$ \\ A, Research Scholar, Bharathiar University Coimbatore - 641 046, Tamil Nadu, INDIA \\ ${ }^{b}$ Department of Mathematics,Justice Basheer Ahmed Sayeed College For Women, Chennai - 600018, Tamil Nadu, INDIA \\ sharmi.beermohamed@gmail.com
}

Article History: Received: 11 January 2021; Accepted: 27 February 2021; Published online: 5 April 2021

Abstract: Let $\mathrm{G}$ be a simple graph of order $\mathrm{n}$ with vertex set $\mathrm{V}=\left\{\mathrm{v}_{1}, \mathrm{v}_{2}, \ldots, \mathrm{v}_{\mathrm{n}}\right\}$ and edge set $\mathrm{E}=\left\{\mathrm{e}_{1}, \mathrm{e}_{2}, \ldots, \mathrm{e}_{\mathrm{m}}\right\}$. A subset of $\mathrm{E}$ is called an edge dominating set of $\mathrm{G}$ if every edge of $\mathrm{E}-\quad$ is adjacent to some edge in .Any edge dominating set with minimum cardinality is called a minimum edge dominating set [2]. Let be a minimum edge dominating set of a graph $\mathrm{G}$. The minimum edge dominating matrix of $\mathrm{G}$ is the $\mathrm{m} \mathrm{x}$ m matrix defined by

$$
D^{\prime}\left({ }^{i} \mathrm{G}\right)=\left(d ^ { \prime } \text { , where } \quad \left(d^{\prime}=\left\{\begin{array}{rr}
1 \text { if } e_{i} \text { ande } e_{j} \text { are incide } \\
1 & \text { if } i=j \text { and } e_{i} \in \\
0 & \text { otherw: }
\end{array}\right.\right.\right.
$$

The characteristic polynomial of $D^{\prime}$ ('is denoted by

$f_{n}(G, \rho)=\operatorname{det}(\rho I-\quad(G))$.

The minimum edge dominating eigen values of a graph $G$ are the eigen values of $(G)$. Minimum edge dominating energy of $\mathrm{G}$ is defined as

$$
l_{(\mathrm{G})}=\sum_{i=1}^{m} l_{[12]}
$$

In this paper we have computed the Minimum Edge Dominating Energy of a graph. Its properties and bounds are discussed. All graphs considered here are simple, finite and undirected.

Key Words:Edge Adjacency Matrix, Edge Energy, Edge Dominating set, Minimum Edge Dominating Eigen values, Minimum Edge Dominating Energy

\section{Introduction}

Euler's work on Konigsberg bridge problem in 1736 paved the way to a new branch of Mathematics called Graph theory. In the year 1978, Ivan Gutman [5] introduced the concept of energy of a graph. The various upper and lower bounds for energy of a graph have been found $[4,6]$.

Recently the interest in graph energy has increased and various energies have been introduced and their properties were discussed. Adiga. C, Bayad. A, Gutman .I, Srinivas .S. A, has introduced a new energy Minimum covering energy of a graph and its properties were dicussed [1]. Recently Rajesh Kanna. M. R, Dharmendra. B. N, Sridhara .G introduced the minimum dominating energy of a graph which depends on the minimum dominating set [11]. The concept of edge domination was introduced by Mitchell and Hedetniemi [10]. Meenakshi. S, Lavanya. S has introduced a new energy Minimum Dom Strong Dominating Energy and its properties and bounds were found [9].

Motivated by these papers, we have introduced the Minimum Edge Dominating Energy of a graph [12]. In this paper we are concerned with finite, simple and undirected graphs. In this paper we have computed the Minimum Edge Dominating Energy of a graph. Its properties and bounds are discussed.

\section{PRELIMINARIES}

Definition: 2.1

The adjacency matrix $\mathrm{A}(\mathrm{G})$ of a graph $\mathrm{G}(\mathrm{V}, \mathrm{E})$ with a vertex set $\left\{\mathrm{e}_{1}, \mathrm{e}_{2}, \ldots, \mathrm{e}_{\mathrm{m}}\right\}$ is an $\mathrm{n} \mathrm{x}$ matrix

$$
\mathrm{A}=\left(\mathrm{a}_{\mathrm{ij}}\right)=\left\{\begin{array}{c}
1 \text { if } v_{i} \text { is adjacent to } \\
0 \text {, otherwise }
\end{array}\right.
$$

$\mathrm{A}$ is a real symmetric matrix.

\section{Definition: 2.2}


The Eigen values $\lambda_{1}, \lambda_{2}, \ldots, \lambda_{n}$ of $A$, assumed in non increasing order, are the Eigen values of the graph G. As A is real symmetric, the Eigen values of $G$ are real with sum equal to zero. The Energy $E(G)$ of $G$ is defined to be the sum of the absolute values of the Eigen values of $\mathrm{G}$.

$$
\text { i.e., } \quad \mathrm{E}(\mathrm{G})=\sum_{i=1}^{n} \|_{i}[5] \text {. }
$$

\section{Definition: $\mathbf{2 . 3}$}

Let $G$ be a simple graph of order $n$ with vertex set $V=\left\{v_{1}, v_{2}, . ., v_{n}\right\}$ and edge set $E=\left\{e_{1}, e_{2}, \ldots, e_{m}\right\}$. A subset of $\mathrm{E}$ is called an Edge Dominating set of $\mathrm{G}$ if every edge of $\mathrm{E}$ - $\quad$ is adjacent to some edge in . Any edge dominating set with minimum cardinality is called a Minimum Edge Dominating Set [10]. Let be a Minimum Edge Dominating Set of a graph G. The Minimum Edge Dominating Matrix of G is the $\mathrm{m}$ x $\mathrm{m}$ matrix defined by

$$
D^{\prime}(=) \quad\left(d ^ { \prime } , \text { where } \quad \left(d^{\prime}=\left\{\begin{array}{rr}
1 \text { if } e_{i} \text { and } e_{j} \text { are adjace } \\
1 & \text { if } i=j \text { and } e_{i} \in i \\
0 & \text { otherwi }
\end{array}\right.\right.\right.
$$

The characteristic polynomial of $D^{\prime}\left({ }^{\prime}\right.$ is denoted by

$$
\mathrm{f}_{\mathrm{m}}(\mathrm{G}, \rho)=\operatorname{det}(\rho \mathrm{I}-\quad(\mathrm{G})) \text {. }
$$

The Minimum Edge Dominating Eigen values of a graph $G$ are the eigen values $\rho_{1}, \rho_{2}, \ldots, \rho_{\mathrm{m}}$ of (G). Minimum Edge Dominating Energy of $G$ is defined as

$$
E_{(\mathrm{G})}=\sum_{i=1}^{m} l_{[12]}
$$

\section{Example: 1}

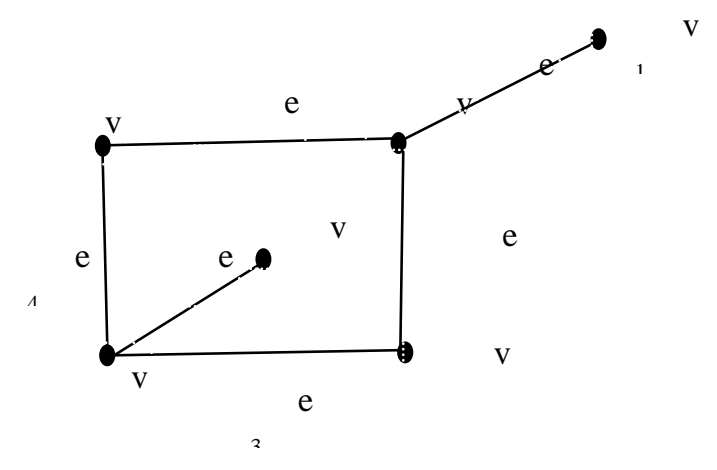

Figure 1

Consider the above graph $\mathrm{G}$.

(i) Let the Minimum Edge Dominating set be $=\left\{\mathrm{e}_{1}, \mathrm{e}_{3}\right\}$.

Then the Minimum Edge Dominating adjacency matrix is

$$
(\mathrm{G})=\left[\begin{array}{lllll}
1 & 1 & 0 & 0 & 1 \\
1 & 0 & 1 & 0 & 1 \\
0 & 1 & 1 & 1 & 0 \\
0 & 0 & 1 & 0 & 1 \\
1 & 1 & 0 & 1 & 0 \\
0 & 0 & 1 & 1 & 0
\end{array}\right.
$$

The characteristic equation is $\rho^{6}-2 \rho^{5}-7 \rho^{4}+7 \rho^{3}+13 \rho^{2}-0 \rho-1=0$.

The Minimum Edge Dominating eigen values are $\rho_{1} \approx-1.8363, \rho_{2} \approx-1.1157, \rho_{3} \approx-0.3132, \rho_{4} \approx 0.2642, \rho_{5} \approx 1.9050, \rho_{6} \approx 3.0962$.

The Minimum Edge Dominating Energy, $\mathrm{E}_{\mathrm{D}}(\mathrm{G}) \approx 8.5306$.

(ii) If we take another Minimum Edge Dominating set $\quad=\left\{\mathrm{e}_{2}, \mathrm{e}_{3}\right\}$. 


$$
(\mathrm{G})=\left[\begin{array}{lllll}
0 & 1 & 0 & 0 & 1 \\
1 & 1 & 1 & 0 & 1 \\
0 & 1 & 1 & 1 & 0 \\
0 & 0 & 1 & 0 & 1 \\
1 & 1 & 0 & 1 & 0 \\
0 & 0 & 1 & 1 & 0
\end{array}\right.
$$

The characteristic equation is $\rho^{6}-2 \rho^{5}-7 \rho^{4}+6 \rho^{3}+13 \rho^{2}-0 \rho-3=0$

The Minimum Edge Dominating Eigen values are

$$
\rho_{1} \approx-1.7321, \rho_{2} \approx-1, \rho_{3} \approx-0.6751, \rho_{4} \approx 0.4608, \rho_{5} \approx 1.7321, \rho_{6} \approx 3.2143
$$

The Minimum Edge Dominating Energy, $\mathrm{E}_{\mathrm{D}}$ ' $(\mathrm{G}) \approx 8.8144$.

This example illustrates the fact that the Minimum Edge Dominating Energy of a graph G depends on the choice of the Minimum Edge Dominating Set.

i.e. The Minimum Edge Dominating Energy is not a graph invariant.

\section{PROPERTIES OF MINIMUM EDGE DOMINATING ENERGY:}

\section{Theorem: 3.1}

Let $\mathrm{G}$ be a simple graph of order $\mathrm{n}$ and size $\mathrm{m}$, let be the Minimum Edge Dominating Set and let $\mathrm{f}_{\mathrm{m}}(\mathrm{G}, \rho)=$ $\mathrm{c}_{0} \rho^{\mathrm{m}}+\mathrm{c}_{1} \rho^{\mathrm{m}-1}+\mathrm{c}_{2} \rho^{\mathrm{m}-2}+\ldots \ldots+\mathrm{c}_{\mathrm{m}}$ be the characteristic polynomial of the Minimum Edge Dominating Matrix of the graph G. Then

\section{Proof:}

$$
c_{2}=\left(\begin{array}{c}
\|_{D s} \\
2
\end{array}-\sum_{i=1}^{m}\left(\begin{array}{c}
\text { deg } \\
2
\end{array} .\right.\right.
$$

The sum of the determinants of all $2 \times 2$ principal sub matrices of $\quad(G)=(-1)^{2} c_{2}$.

$$
\begin{aligned}
& \text { Therefore, } c_{2}=\sum_{1 \leq i<j \leq m} \mid \begin{array}{ll}
d_{i i}^{\prime} & d^{i} \\
d_{j i}^{\prime} & d^{i}
\end{array} \\
& \left.=\sum_{1 \leq i<j \leq} d_{i i}^{\prime} c_{-} d_{i j}^{\prime}\right)^{\prime} \\
& =\sum_{1 \leq i<j \leq m} d_{i i}^{\prime} c-\sum_{1 \leq i<j \leq m} c \\
& =\left(\begin{array}{c}
\|_{i} \\
2
\end{array}-\sum_{i=1}^{m}\left(\begin{array}{c}
\mathrm{deg} \\
2
\end{array}\right. \text {. }\right.
\end{aligned}
$$

\section{Theorem: 3.2}

Let $\mathrm{G}=(\mathrm{V}, \mathrm{E})$ be a simple graph of order $\mathrm{n}$ and size $\mathrm{m}$. Let $\rho_{1}, \rho_{2}, \rho_{1}, \ldots \ldots, \rho_{\mathrm{m}}$ be the eigen values of

Then $\quad \sum_{i=1}^{m} l=\mid L_{+} 2\left[\sum_{i=1}^{m}\left(\begin{array}{c}\operatorname{deg} v_{i} \\ 2\end{array}\right.\right.$.

Proof:

The sum of the squares of the eigen values of

$$
D^{\prime}\left(\text { is the trace of } \quad \left[D^{\prime}(G)\right.\right. \text {. }
$$

Therefore, $\quad \sum_{i=1}^{m} l=\sum_{i=1}^{m} \sum_{j=1}^{m} d_{i j}^{v}$

$$
\begin{aligned}
= & \sum_{i=1}^{m} c_{+} \sum_{i \neq j} d^{\prime}{ }_{i j}^{c} \\
& =\sum_{i=1}^{m} d_{+} 2 \sum_{i<j} c \\
& =D_{+} 2\left[\sum _ { i = 1 } ^ { m } \left(\begin{array}{c}
\operatorname{deg} v \\
2
\end{array}\right.\right.
\end{aligned}
$$

\section{BOUNDS FOR MINIMUM EDGE DOMINATING ENERGY}

In this section we find some bounds for

$E_{(\mathrm{G})}$ of a graph.

Theorem: 4.1 
Let $\mathrm{G}$ be a simple graph with $\mathrm{n}$ vertices and m edges. If $E_{(\mathrm{G})}$ is the Minimum Edge Dominating Energy of the graph, then

\section{Proof:}

$$
\sqrt{\left|D^{\prime}\right|+2\left[\sum _ { i = 1 } ^ { m } \left(\operatorname{deg}_{2}\right.\right.} \leq E_{(G)} \leq \sqrt{m\left[\left|D^{\prime}\right|+2\left[\sum _ { i = 1 } ^ { m } \left(\begin{array}{c}
\text { deg } v_{i}^{i} \\
2
\end{array}\right.\right.\right.}
$$

Consider the Cauchy-Schwartz inequality

$$
\begin{aligned}
& \left(\sum _ { i = 1 } ^ { n } a _ { i } b \quad ( \sum _ { i = 1 } ^ { n } a _ { i } ^ { 2 } ) \left(\sum_{i=1}^{n} b_{i}\right.\right. \\
& \text { If } \quad=1, \quad=|\quad|, \mathrm{i}=1, \ldots, \mathrm{m} \\
& \text { Then, } \quad\left(\sum_{i=1}^{m} \mid \rho_{i} \quad\left(\sum_{i=1}^{m}\right] \quad\left(\sum_{i=1}^{m} \mid \rho_{i}\right.\right. \\
& \left(E _ { D y } \left(\mathrm { G } _ { i } \quad \mathrm { m } \quad \left[\left|D^{\prime}\right|+2\left[\sum_{i=1}^{m}\left(\begin{array}{c}
\operatorname{deg} v_{i} \\
2
\end{array}\right)\right.\right.\right.\right.
\end{aligned}
$$

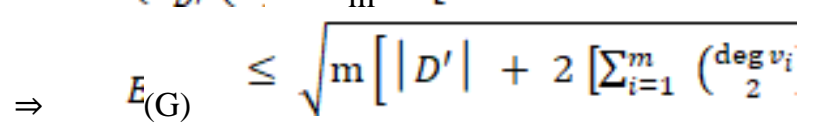

Therefore, the upper bound holds. For the lower bound, since

Similar to Mc Clellands [8] bounds for energy of a graph, bounds for $E_{(G)}$ are given in the following theorem.

\section{Theorem: 4.2}

Let $\mathrm{G}$ be a simple graph with $\mathrm{n}$ vertices and m edges. If $E_{(\mathrm{G})}$ is the Minimum Edge Dominating Energy of the graph and let $\mathrm{P} \quad{ }^{\prime}=\operatorname{det}\left(D^{\prime}(C\right.$, then

Proof:

$$
\begin{aligned}
& \begin{array}{ll}
\left(\sum_{i=1}^{m}\left|\rho_{i}\right| \geq \sum_{i=1}^{m} /\right. \\
\quad\left(E_{D y}(\mathrm{G}) \quad \mid L_{+} 2\left[\sum_{i=1}^{m}\left(\begin{array}{c}
\operatorname{deg} v \\
2
\end{array}\right)\right.\right.
\end{array} \\
& \Rightarrow \quad E_{(\mathrm{G})} \quad \sqrt{\left|D^{\prime}\right|+2\left[\sum _ { i = 1 } ^ { m } \left(\operatorname{deg}_{2}\right.\right.} \\
& \sqrt{\left|D^{\prime}\right|+2\left[\sum _ { i = 1 } ^ { m } \left(\operatorname{deg}_{2}\right.\right.} \leq E_{(\mathrm{G})} \leq \sqrt{\mathrm{m}\left[\left|D^{\prime}\right|+2\left[\sum _ { i = 1 } ^ { m } \left(\begin{array}{c}
\operatorname{deg} v_{i}^{\prime} \\
2
\end{array}\right.\right.\right.}
\end{aligned}
$$

From the relation between the arithmetic mean and geometric mean, we have

$$
\begin{aligned}
\frac{1}{m(m-1)} \sum_{i \neq j} \mid f & \rho \quad\left[\prod_{i \neq j}\left|\rho_{i}\right|\left|\rho_{j}\right|\right]^{\frac{1}{m[m}} \\
= & {\left[\prod_{i=1}^{m}\left|\rho_{i}\right|^{2(m-1)}\right]^{\frac{1}{m[m}} } \\
& =\left[\prod_{i=1}^{m} \rho_{i}\right. \\
& =\operatorname{det}\left(D^{\prime}(G)\right. \\
= & \mathrm{F}
\end{aligned}
$$

Therefore,

$$
\sum_{i \neq j}|f \quad| \rho \quad \geq m(m-1) \mathrm{F}
$$

Now consider,

$$
\begin{aligned}
\left(E_{D^{\prime}}(\mathrm{G})=\right. & \left(\sum_{i=1}^{m} \mid \rho_{i}\right. \\
= & \sum_{i=1}^{m}\left|\rho_{+} \quad \sum_{i \neq j}\right| \rho_{i} \|_{i} \\
& \geq \sum_{i=1}^{m}\left|\rho_{i}\right|^{2}+m(m-1) \mathrm{I} \\
= & {\left[\left|D^{\prime}\right|+2\left[\sum_{i=1}^{m}\left(\operatorname{deg}_{2} v_{i}\right)_{+} \quad m(m-1) \mathrm{I}\right.\right.}
\end{aligned}
$$

[Theorem: 3.1] 


$$
\therefore \quad E_{(\mathrm{G})} \quad \sqrt{\left[\left|D^{\prime}\right|+2\left[\sum_{i=1}^{m}\left(\operatorname{deg}_{2} v_{i}\right)\right]\right]+\mathrm{m}(\mathrm{m}-1) \mathrm{H}}
$$

\section{Theorem: 4.3}

If $\rho_{1}$ (is the largest Minimum Edge Dominating Eigen value of $(\mathrm{G})$, then

$$
\rho_{1}(G) \geq \frac{\left\|D^{\prime}\right\|+2\left[\sum _ { i = 1 } ^ { m } \left(\begin{array}{c}
\operatorname{deg}_{2} \\
m
\end{array}\right.\right.}{m} .
$$

Proof:

Let $\mathrm{X}$ be any non zero vector. Then by [3], we have

Therefore,

$$
\rho_{1}(G)=\max _{X \neq 0}\left\{\frac{X \prime D}{X s^{\prime}}\right.
$$

$$
\rho_{1}(G) \geq \frac{F D V J}{J I}=\frac{\|D r\|+2\left[\sum _ { i = 1 } ^ { m } \left(\begin{array}{c}
\text { deg } \\
2
\end{array}\right.\right.}{m},
$$

where $\mathrm{J}=[1,1,1, \ldots . .1]^{\prime}$ is a unit column matrix of order $\mathrm{m} \mathrm{x} 1$.

Similar to Koolen and Moulton's [7] upper bound for energy of a graph, upper bound for $\quad E_{(G)}$, is given in the following theorem.

\section{Theorem: 4.4}

If $\mathrm{G}$ is a simple graph with $\mathrm{n}$ vertices and m edges and $\quad \mid D_{+} \quad 2\left[\sum_{i=1}^{m}\left(\underset{2}{\operatorname{deg} v_{i}}\right)\right] \geq$ then

$$
\begin{aligned}
& E_{D^{\prime}}(G)
\end{aligned}
$$

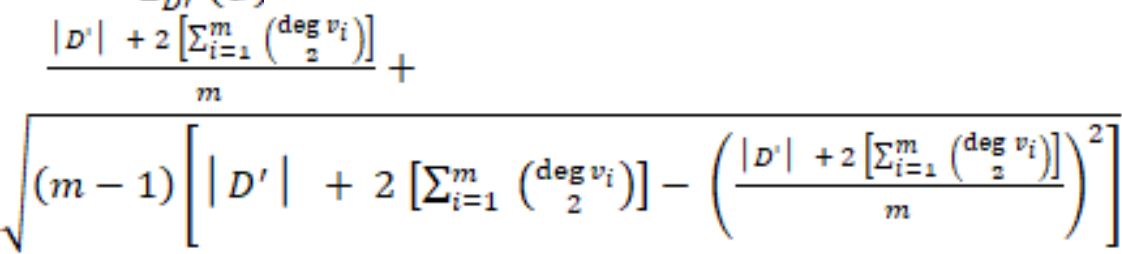

\section{Proof:}

Consider the Cauchy-Schwartz inequality

$$
\begin{aligned}
& \left(\sum _ { i = 2 } ^ { n } a _ { i } b \quad ( \sum _ { i = 2 } ^ { n } a _ { i } ^ { 2 } ) \left(\sum_{i=2}^{n} b_{i}\right.\right. \\
& \begin{array}{l}
\text { If } \quad=1, \quad=|\quad|, \mathrm{i}=2, \ldots, \mathrm{m} \\
\text { Then, } \quad\left(\sum_{i=2}^{m} \mid \rho_{i} \quad\left(\sum _ { i = 2 } ^ { m } 1 \quad \left(\sum_{i=2}^{m} \mid \rho_{i}\right.\right.\right.
\end{array} \\
& \begin{array}{ll}
\text { Then, } \quad\left(\sum_{i=2}^{m} \mid \rho_{i} \quad\left(\sum_{i=2}^{m}\right] \quad\left(\sum_{i=2}^{m} \mid \rho_{i}\right.\right. \\
\Rightarrow \quad\left[E_{D,}(G)-\rho_{1}\right]^{2} \leq(m-1)\left[\left|D^{\prime}\right|+2\left[\sum_{i=1}^{m}\left(\underset{2}{\operatorname{deg} v_{i}}\right)\right]-\rho .\right.
\end{array} \\
& \Rightarrow \quad E_{D^{\prime}}(G) \leq \rho_{1}+\sqrt{(m-1)\left[\left|D^{\prime}\right|+2\left[\sum_{i=1}^{m}\left(\underset{2}{\operatorname{deg} v_{i}}\right)\right]-\rho\right.} . \\
& f\left(=x+\sqrt{(m-1)\left[\left|D^{\prime}\right|+2\left[\sum_{i=1}^{m}\left(\operatorname{deg}_{2} v_{i}\right)\right]-3\right.}\right.
\end{aligned}
$$

For decreasing function

$$
\begin{aligned}
& f^{\prime} \quad 0 \Rightarrow 1-\frac{(m-1) x}{\sqrt{(m-1)\left[\left|D^{r}\right|+2\left[\sum_{i=1}^{m}\left({ }^{\operatorname{deg} N_{i}}\right)\right]-x^{2}\right]}} \leq \\
& \Rightarrow \quad x \geq \sqrt{\frac{\|D r\|+2\left[\sum _ { i = \mathbb { 1 } } ^ { m } \left(\begin{array}{c}
\mathrm{deg} \\
m
\end{array}\right.\right.}{m}} \\
& \text { Since } \quad \mid D_{+} 2\left[\sum_{i=1}^{m}\left(\begin{array}{c}
\operatorname{deg} v_{i} \\
2
\end{array}\right)\right] \geq \text {, }
\end{aligned}
$$

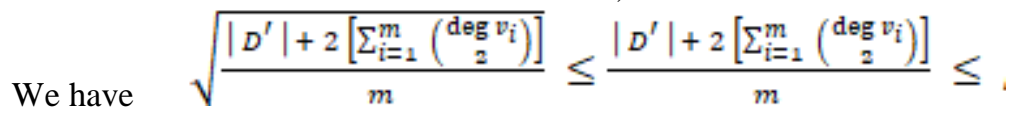$$
\therefore \quad f\left(\rho_{1}\right) \leq f\left(\frac{\left|D^{t}\right|+2\left[\sum _ { i = 1 } ^ { m } \left({ }_{a}^{\operatorname{deg} w_{i}}\right.\right.}{m}\right.
$$ 


$$
\begin{aligned}
& \Rightarrow \quad E_{D,}(G) \leq f\left(\rho_{1}\right) \leq f\left(\frac{\left|D^{\prime}\right|+2\left[\sum _ { i = 1 } ^ { m } \left(\operatorname{deg}_{z} w_{i}\right.\right.}{m}\right. \\
& \Rightarrow \quad E_{D^{\prime}}(G) \leq f\left(\frac{\left\|D^{r}\right\|+2\left[\sum _ { i = 1 } ^ { m } \left(\operatorname{deg}_{z} w_{i}\right.\right.}{m}\right. \\
& \therefore E_{D s}(G)
\end{aligned}
$$

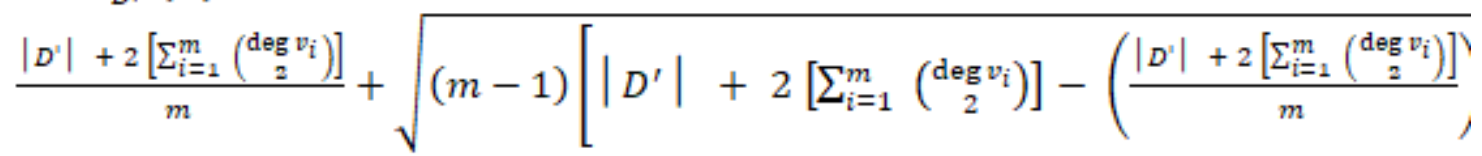

\section{CONCLUSION:}

In this paper we have found the Minimum Edge Dominating energy of a graph. The various upper and lower bounds for the Minimum Edge Dominating Energy of a graph have been found. Analogues works can be carried by us for other graphs also.

\section{REFERENCES}

1. Adiga. C, Bayad. A, Gutman .I, Srinivas .S.A, The minimum covering energy of a graph, Kragujevac J. Sci.34, 39-56 (2012).

2. Arumugam. S, Velammal. S, Edge domination in graphs, Taiwanese Journal of Mathematics, vol. 2, no. 2, 173-179 (1998).

3. Bapat. R.B, Graphs and Matrices, Hindustan Book Agency, P.32, (2011).

4. Ch. Das. K. and Gutman. I, Bounds for the energy of graphs, Hacettepe Journal of Mathematics and Statistics 45(3), 695-703 (2016).

5. Gutman. I, The energy of a graph, Ber. Math-Satist. Sekt. Forschungsz.Graz103, 1-22 (1978).

6. Jahanbani. A, Lower bounds for the energy of graphs, AKCE International Journal of Graphs and Combinatorics, vol. 15, issue 1, 88-96 (2017).

7. Koolen. J.H, Moulton. V, Maximal energy graphs, Adv.Appl. Math. 26, 47 - 52 (2001).

8. Mc Clelland. B.J, Properties of the Latent Roots of a Matrix: The Estimation of $\pi$-Electron Energies, The Journal of Chemical Physics, vol. 54, issue 2, p.640-643 (1971).

9. Meenakshi. S, Lavanya. S, Minimum Dom Strong Dominating Energy of Graph, International Journal of Pure and Applied Mathematics, vol. 115, 1039-1047(2017).

Mitchell. S, Hedetniemi. S. T, Edge domination in trees, Congr. Numer19, 489-509 (1977).

10. Rajesh Kanna. M. R, Dharmendra. B. N, Sridhara. G, The Minimum Dominating Energy Of A Graph, International Journal of Pure and Applied Mathematics vol. 85 No. 4, 707-718 (2013).

11. Sharmila. A, Lavanya. S, The minimum edge dominating energy of a graph, Journal of Computer and Mathematical Sciences, vol. 8, 824-828 (2017). 